\title{
ANTECEDENTES DEL CRECIMIENTO DE Acacia mearnsii De Wild SEGÚN DISTINTAS DENSIDADES INICIALES PARA DOS SITIOS EN LA REGIÓN DEL BIO BIO
}

\author{
Pinilla, Juan Carlos ${ }^{11}$ y Navarrete, Mauricio ${ }^{12}$
}

\section{RESUMEN}

Las plantaciones forestales en Chile alcanzan ya a una superficie total de más de 2 millones de hectáreas y son la base de una desarrollada y creciente industria forestal, sin embargo están concentradas fuertemente en las regiones de Maule, Bio Bio y La Araucanía, y están muy mayoritariamente compuestas por tres especies; Pinus radiata, Eucalyptus globulus y Eucalyptus nitens, que en conjunto representan el $93 \%$ de la superficie total plantada.

Dentro de las regiones mencionadas y en otras regiones del país existen aún importantes superficies de suelos forestales desarbolados y degradados, que requieren de una cubierta forestal que los recupere y los incorpore a la producción, hacia los cuales se puede ampliar considerablemente la superficie de plantaciones forestales del país con los beneficios económicos, sociales y ambientales que esto involucraría.

El Instituto Forestal (INFOR) desarrolla diferentes experiencias con especies exóticas de rápido crecimiento, apropiadas para la forestación en el país y para diversificar las especies empleadas en las plantaciones forestales y los productos a obtener de estas, con especial énfasis en zonas que hoy resultan marginales para estas últimas debido a limitantes de sitio como frío, restricciones hídricas u otras.

Diversas líneas de trabajo de INFOR con los fines mencionados han incluido especies del género Acacia, como Acacia dealbata, A. melanoxylon, A. mearnsii y A. saligna, especies originarias de Australia que son de rápido crecimiento, se adaptan a variadas condiciones de sitio en el país y en términos productivos son consideradas multipropósito.

En el presente trabajo se entrega resultados de dos ensayos de espaciamiento de plantación con Acacia mearnsii, especie que INFOR introdujo al país en los años 80 del siglo pasado, establecidos en el año 2004 en dos diferentes sitios de la región del Bio Bio.

Los resultados a 8 años de edad confirman a la especie como una alternativa interesante para la forestación con distintos fines y en cortas rotaciones, y ponen en evidencia la relevancia que tiene el espaciamiento de plantación en función de diferentes productos objeto, como madera sólida, pulpa, energía y otros.

Palabras clase: Espaciamiento de plantación, Acacia mearnsii, Silvicultura.

\footnotetext{
${ }_{11}^{11}$ Instituto Forestal, Chile. jpinilla@infor.cl

12 Instituto Forestal, Chile. mnavarre@infor.cl
} 


\section{SUMMARY}

Planted forests in Chile already cover a total area over than 2 million hectares and are the basis to a developed and increasing forest industry. However, forest plantations are strongly concentrated in the Maule, Bio Bio and La Araucanía regions and are mainly composed by only three species; Pinus radiata, Eucalyptus globulus and Eucalyptus nitens, which as a group represents $93 \%$ of the total planted area.

The mentioned regions and other regions in the country still present deforested and degraded forest soil important areas where planted forests can be considerably expanded with the involved economic, social and environmental benefits.

The Chilean Forestry Institute (INFOR) is developing different research lines with exotic fast growing species, appropriated to afforestation plans and to diversify species and production, with emphasis on zones in the country currently not used because of site limitations, like frost, drought or others.

Several INFOR research lines following the mentioned objectives have included Acacia genus species, as Acacia dealbata, A. melanoxylon, $A$. mearnsii and $A$. saligna, which are Australian fast growing species and have already shown a good adaptation to different zone in Chile and can offer a variety of products.

The present paper reviews results from plantation density trials with Acacia mearnsii, introduced to the country by INFOR by the 80 s of the last century, established in 2004 in two different sites of the Bio Bio region.

Results at 8 year old confirm the species as an interesting alternative for short rotation afforestation plans and highlight the importance of plantation density in function of different production objectives, as solid wood, pulp, energy and others.

Key words: Plantation density, Acacia mearnsii, Silviculture 


\section{INTRODUCCIÓN}

En las últimas décadas el sector forestal chileno ha experimentado una fuerte expansión de las plantaciones de especies exóticas de rápido crecimiento, especialmente Pinus radiata $D$. Don. y Eucalyptus globulus Labill. y más recientemente Eucalyptus nitens (Deane and Maiden) Maiden.

Sin embargo, pese a que las plantaciones forestales en el país sobrepasan ya largamente los 2 millones de hectáreas, aún existen importantes superficies de suelos forestales degradados o sin uso, en los que el cultivo de pino o de eucalipto no ha tenido buenos resultados, y se hace necesaria la diversificación de las especies forestales para repoblar estos terrenos, incorporarlos a la producción y diversificar esta, atenuar potenciales problemas fitosanitarios y promover un mayor desarrollo rural.

Las investigaciones del Instituto Forestal (INFOR) en esta área apuntan a generar, evaluar y difundir información sobre el estado y desarrollo de plantaciones forestales, en aspectos relacionados a su productividad, desarrollo, manejo, potencial económico de cultivo, sanidad e identificación de nuevas opciones productivas, aspectos considerados estratégicos para incrementar la competitividad sectorial.

Diferentes especies de acacias se están ya usando incipientemente en la forestación en el país, pero para incrementar su uso se requiere conocer los parámetros básicos de crecimiento, productividad y aprovechamiento industrial, lo cual propiciaría la creación de nuevos recursos forestales con ellas más allá de la áreas habituales de plantaciones en el país y la consolidación de mercados para sus productos.

Algunas de las investigaciones de INFOR en la materia indican que especies del género Acacia que crecen en Chile; como Acacia dealbata Link, Acacia melanoxylon R. Br. y Acacia mearnsii De Wild., en la zona centro sur, y Acacia saligna, en la zona centro norte, han respondido bien a las condiciones edafoclimáticas locales, desarrollándose con rapidez y ofreciendo una variedad de usos, lo que las convierte en un recurso económicamente interesante de aprovechar.

Estas características de rápido crecimiento y adaptación a diversos sitios han constituido a estas especies en buenas alternativas también para pequeños y medianos propietarios, quienes podrían obtener ingresos a partir de rodales establecidos en sus predios.

INFOR ha instalado, medido y analizado diversos ensayos para el estudio del crecimiento y rendimiento de acacias con el objetivo de determinar respuestas a diferentes espaciamientos iniciales y esquemas de manejo factibles de utilizar en una mayor escala. Entre estos ensayos destacan parcelas permanentes, ensayos de procedencias, unidades demostrativas y ensayos de espaciamiento.

En el presente trabajo se revisa los resultados de ensayos de espaciamiento de Acacia mearnsii, consistentes en diversas densidades de plantación, dispuestos bajo un diseño experimental determinado y conducentes a la determinación de diferencias significativas, bajo los tradicionales análisis de varianza.

En este tipo de ensayos comúnmente las variables corresponden a volúmenes, diámetros o alturas, o una combinación de estas, que son las variables de interés para el manejo de las plantaciones, y se resume en las varianzas la respuesta integral del tratamiento.

Se presenta los resultados de las evaluaciones de 2 ensayos de espaciamiento con Acacia mearnsii instalados en la Región del Bio Bio durante el año 2004, información que se espera ayude a la definición de un conjunto de regímenes de manejo, específicos por producto o combinación de productos a obtener y eficientes desde el punto de vista económico. 


\section{ANTECEDENTES GENERALES DE Acacia mearnsii}

Acacia mearnsii De Wild. es originaria del sudeste de Australia, se encuentra principalmente en tierras bajas y costeras y en laderas bajas de las mesetas y cordilleras. Se distribuye desde el nivel del mar, hasta aproximadamente $900 \mathrm{msnm}$. Su nombre común, Black Wattle o acacia negra se refiere al follaje y a la corteza, ambos de color oscuro (Pinilla, 2000).

Acacia mearnsii es un arbusto grande o un árbol pequeño (Figura $N^{\circ} 1$ ), comúnmente de 6 a $10 \mathrm{~m}$ de altura, pero puede alcanzar hasta $20 \mathrm{~m}$ (Kannegiesser, 1990). Searle (2000) señala que a los 34 meses esta especie puede alcanzar alturas cercanas a los $6 \mathrm{~m}$ y que bajo condiciones favorables de crecimiento puede a los 40 años llegar a medir $20 \mathrm{~m}$ de altura con más de $50 \mathrm{~cm}$ de DAP. Señala además que esta especie mejora su tamaño y forma en suelos con mayor contenido de humedad.

Los ejemplares que crecen solitarios presentan copas amplias y extendidas, son ramificados desde la base y con el tronco principal torcido. Pero al crecer en plantaciones, el fuste es generalmente recto y delgado hasta tres cuartas partes de la altura total.

La corteza de los árboles viejos es de color café negruzco, dura y fisurada; en individuos más jóvenes y en las partes altas de aquellos de más edad es de color café grisáceo, más delgada y suave.

El follaje adulto es de color verde oscuro con brotes nuevos suavemente amarillos. Su madera es finamente texturada y presenta anillos de crecimiento poco visibles. La albura es de color café muy pálido y el duramen café claro con moteado rojizo. La durabilidad natural es considerada ligera o moderada.

En Australia crece en las zonas climáticas húmedas y subhúmedas, cálidas y frías, con precipitaciones medias anuales de 625 a $900 \mathrm{~mm}$ que caen entre 105 a 175 días al año. Raramente se presenta en zonas donde las temperaturas exceden los $38 \stackrel{\circ}{\circ} \mathrm{C}$ y no crece con temperaturas sobre $40 \stackrel{\circ}{ }$, reportándose áreas en donde ha soportado hasta 40 días con heladas al año (Kannegiesser, 1990).

En cuanto a los suelos, se señala que esta acacia se ha encontrado en basaltos, granitos y areniscas, siendo común en suelos derivados de esquistos y pizarras metamórficas, aluviales y podzoles forestales profundos de moderada a baja fertilidad. En su lugar de origen, los mejores crecimientos se han reportado en suelos húmedos, relativamente profundos, de texturas livianas, bien drenados y generalmente ácidos, con un $\mathrm{pH}$ entre 5 y 6,5. $A$. mearnsii constituye el sotobosque de bosques altos y abiertos, dominados principalmente por Eucalyptus ovata, E. saligna, E. globulus, E. viminalis y E. radiata. En zonas donde coloniza tierras explotadas, suele formar rodales densos (Kannegiesser, 1990; Pinilla, 2000).

En un principio, la madera de esta especie se utilizó como material para construcciones livianas y energía; posteriormente, la corteza se utilizó como curtiente. La especie es una de las fuentes de corteza para la industria de taninos vegetales a nivel mundial, los que son utilizados en la manufactura de cuero duro para zapatos, monturas y otros productos. Las industrias de tanino basadas en esta especie se han desarrollado principalmente en Brasil, Kenya, India, Sudáfrica, Tanzania y Zimbabwe. La corteza presenta entre 36 y $41 \%$ de taninos de muy buena calidad, rendimiento que está en función de factores ambientales (Kannegiesser, 1990).

La literatura reporta otros usos para la corteza y el extracto obtenido, tales como preservantes, adhesivos para molduras de plástico y adhesivos en la industria de la madera prensada para exteriores, tableros de partículas y madera laminada (Campos et al., 1990; Coopens et al., 1980, citados por Kannegiesser, 1990).

Por sus características de rápido crecimiento, adaptabilidad a diversas condiciones de sitio y capacidad colonizadora de áreas erosionadas, esta especie ha sido efectiva para el control de zonas degradadas y para mejorar la fertilidad de suelos, siendo un eficiente fijador de nitrógeno. 
La literatura menciona que en Indonesia se han obtenido 21 a 28 t/año de hojas verdes, conteniendo 240 a $285 \mathrm{~kg}$ de nitrógeno, y que cultivos agrícolas asociados a esta especie rinden el doble gracias a su acción fijadora de nitrógeno (NAS, 1980; Kannegiesser, 1990; Pinilla, 2000).

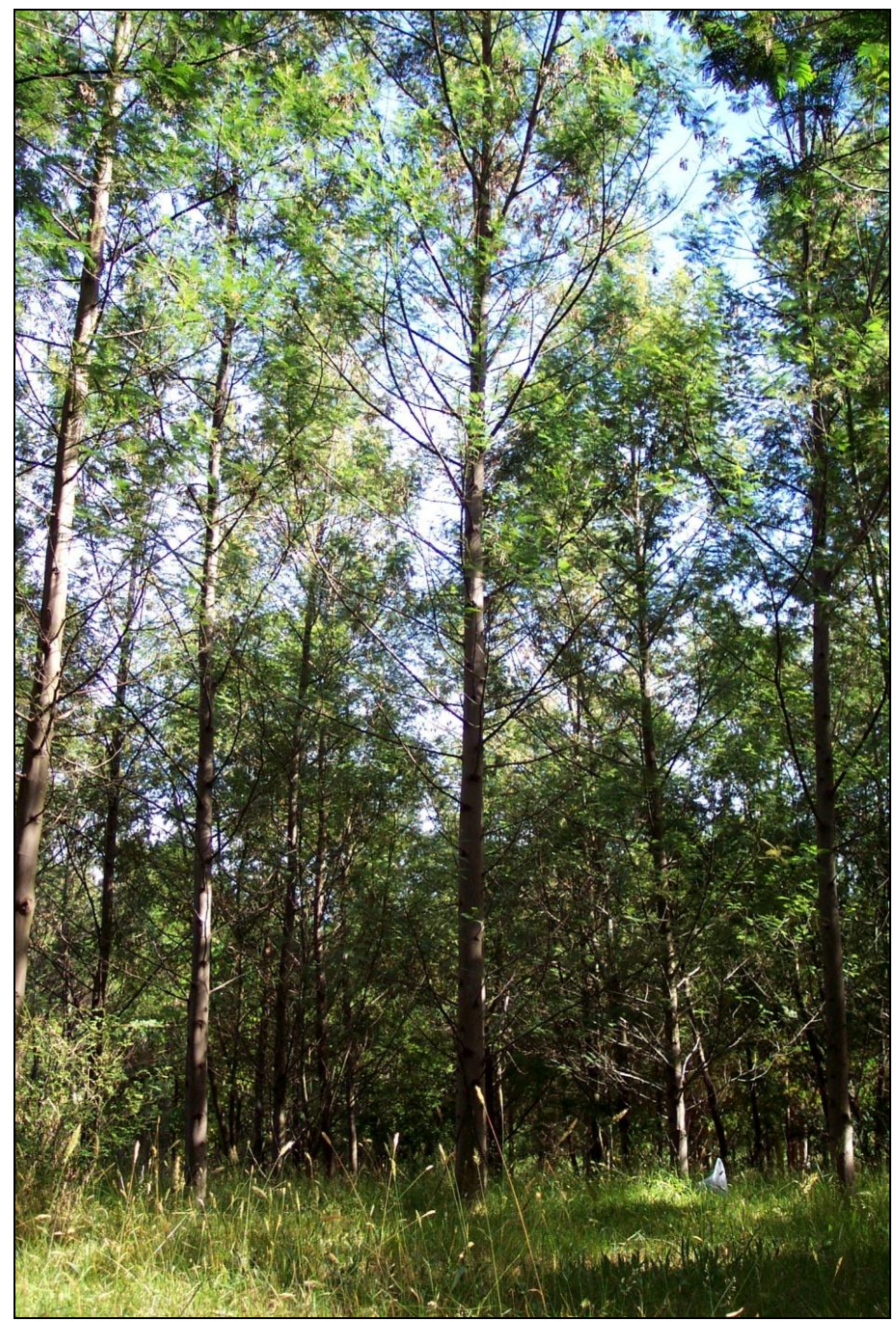

Figura $\mathrm{N}^{\circ} 1$

\section{ÁRBOL ADULTO DE Acacia mearnsii}

En Chile, Acacia mearnsii está recomendada para las regiones de Valparaíso a los Lagos. Su principal producto son los taninos extraíbles de su corteza y la madera de buena calidad para postes. Presenta una moderada resistencia a las heladas y resulta adecuada para la recuperación de sistemas degradados, aplicando técnicas de control de erosión y de restauración de la cubierta vegetacional.

Es posible utilizar esta especie en plantaciones combinadas con especies del género 
Eucalyptus para la producción de fibra corta, tal como se realiza en Australia y Sudáfrica. En Australia, Acacia mearnsii constituye el dosel inferior en bosques naturales de eucaliptos y conforma un porcentaje de la producción de pulpa total debido a su compatibilidad y aptitudes celulósicas (Pinilla, 2000).

Algunos resultados de estudios de pulpaje realizados en Australia con especies latifoliadas de rápido crecimiento han demostrado que Acacia mearnsii presenta características pulpables muy promisorias, lo que se traduce en un alto rendimiento de pulpa kraft, bajos requerimientos de productos químicos y buenas propiedades de resistencia del papel.

En sus áreas de ocurrencia natural, estas acacias juegan un rol importante en la conservación de suelos, como fuente de fijación de nitrógeno en los ecosistemas forestales y como especies forrajeras. Presentan características radiculares que las hacen apropiadas para el control de dunas costeras, para la recuperación de laderas erosionadas y suelos devastados.

Además, la corteza de $A$. mearnsii tiene un alto contenido de taninos, que puede superar el $45 \%$ de la biomasa de la corteza. De acuerdo a NAS (1980) su madera es también apropiada para fines energéticos. Así, su diversidad de posibles usos la transforma en una especie multipropósito.

\section{MATERIAL Y MÉTODO}

Los resultados revisados en el presente trabajo provienen de dos ensayos establecidos por INFOR el año 2004 en la Región del Bio Bio:

Santa Teresa, en Rafael, Comuna de Tomé

San Antonio de Cuda, Comuna de Florida.

\section{Ensayo Santa Teresa, Rafael}

Este ensayo se encuentra ubicado en la Comuna de Tomé, sector Rafael, predio Santa Teresa, y está ubicado en una ladera con exposición Sur-Oeste, aledaño a la Ruta del Itata entre Concepción y Rafael, al lado del Puente Roa.

El ensayo consta de 14 parcelas en 3 bloques con distinto espaciamiento inicial, que va de 1.111 a 2.500 plantas por hectárea, utilizando 49 plantas por parcela. Los tratamientos son los que se indican en el Cuadro $\mathrm{N}^{\circ} 1$.

Cuadro $\mathrm{N}^{\circ} 1$

ESPACIAMIENTO PROBADOS ENSAYOS SANTA TERESA, RAFAEL

\begin{tabular}{|c|c|c|c|}
\hline Tratamiento & $\begin{array}{c}\text { Espaciamiento } \\
(\mathbf{m})\end{array}$ & $\begin{array}{c}\text { Densidad } \\
(\mathbf{a r b} / \mathbf{h a})\end{array}$ & $\begin{array}{c}\text { Repeticiones } \\
\left(\mathbf{N}^{\circ} \mathbf{)}\right.\end{array}$ \\
\hline 1 & $3 \times 1,5$ & 2.222 & 3 \\
\hline 2 & $3 \times 2$ & 1.667 & 3 \\
\hline 3 & $3 \times 3$ & 1.111 & 3 \\
\hline 4 & $2 \times 2$ & 2.500 & 3 \\
\hline 5 & $2 \times 4$ & 1.250 & 2 \\
\hline
\end{tabular}

La parcela de control está dada por las 25 plantas centrales a las cuales se les midió diámetro y altura.

En las Figuras $\mathrm{N}^{\circ} 2$ y $\mathrm{N}^{\circ} 3$ se muestra una visión general del ensayo y su diseño experimental, respectivamente. 


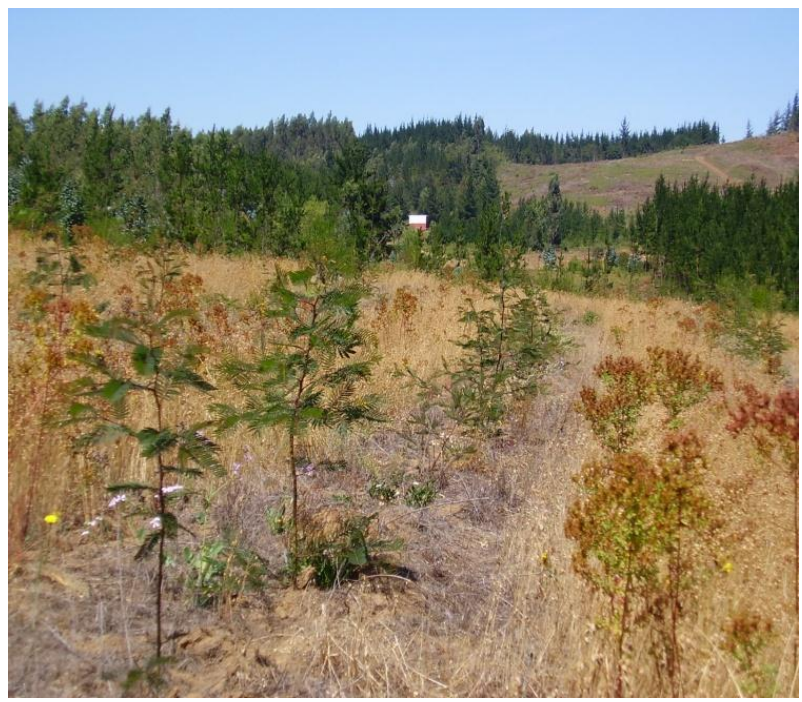

Figura $\mathrm{N}^{\circ} 2$

VISTA GENERAL ENSAYO SANTA TERESA, RAFAEL

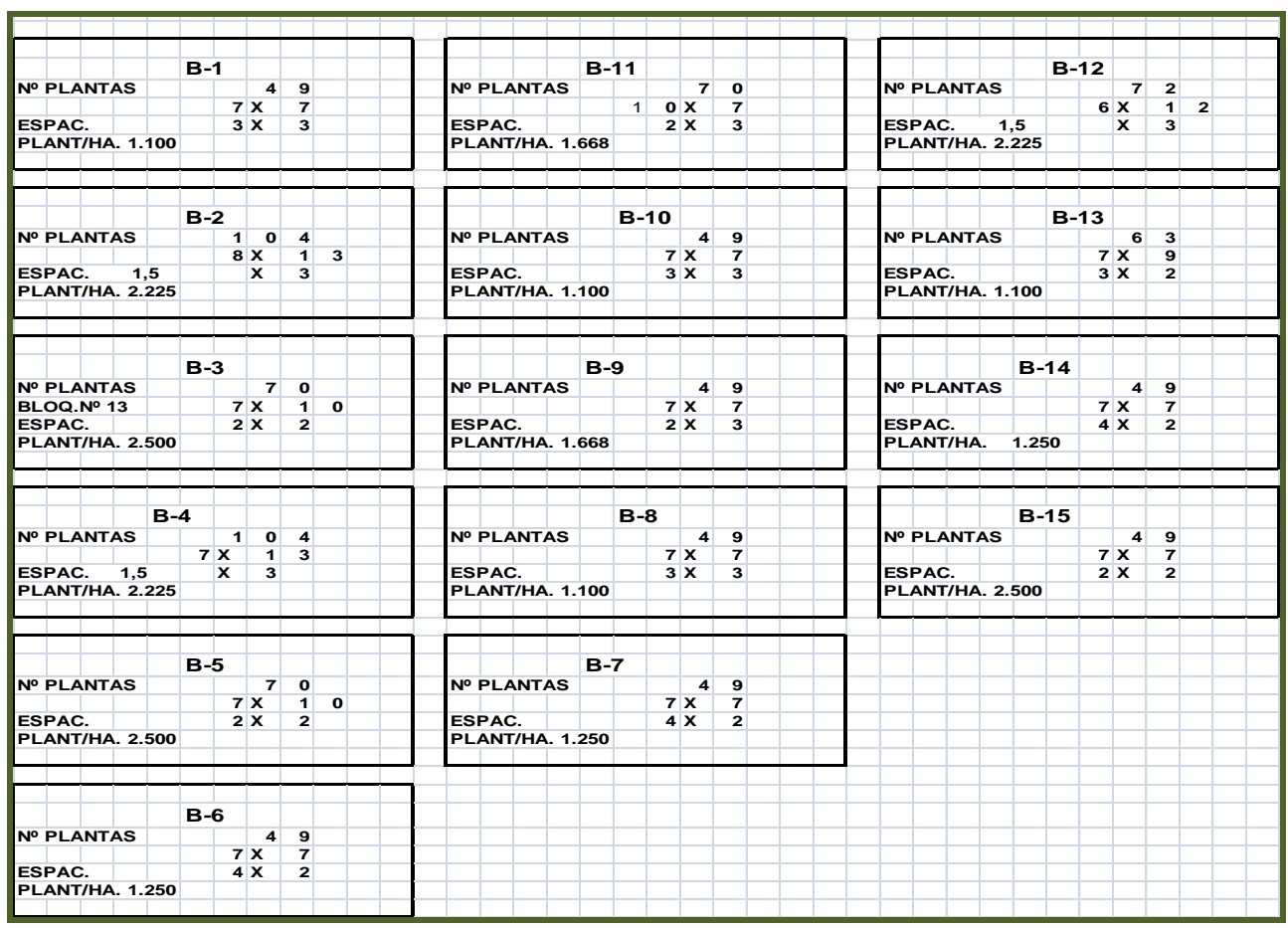

Figura $N^{\circ} 3$

DISEÑO ENSAYO SANTA TERESA, RAFAEL 


\section{Ensayo San Antonio de Cuda, Florida}

Este ensayo se encuentra ubicado en la Comuna de Florida, sector San Antonio de Cuda, $12 \mathrm{~km}$ al norte de Florida, y está instalado en una ladera con exposición Sur.

Tratamientos, diseño, plantas por parcela, parcela de control y variables medidas igual que ensayo anterior.

En las Figuras $\mathrm{N}^{\circ} 4$ y $\mathrm{N}^{\circ} 5$ se muestra una visión general del ensayo y su diseño experimental, respectivamente.
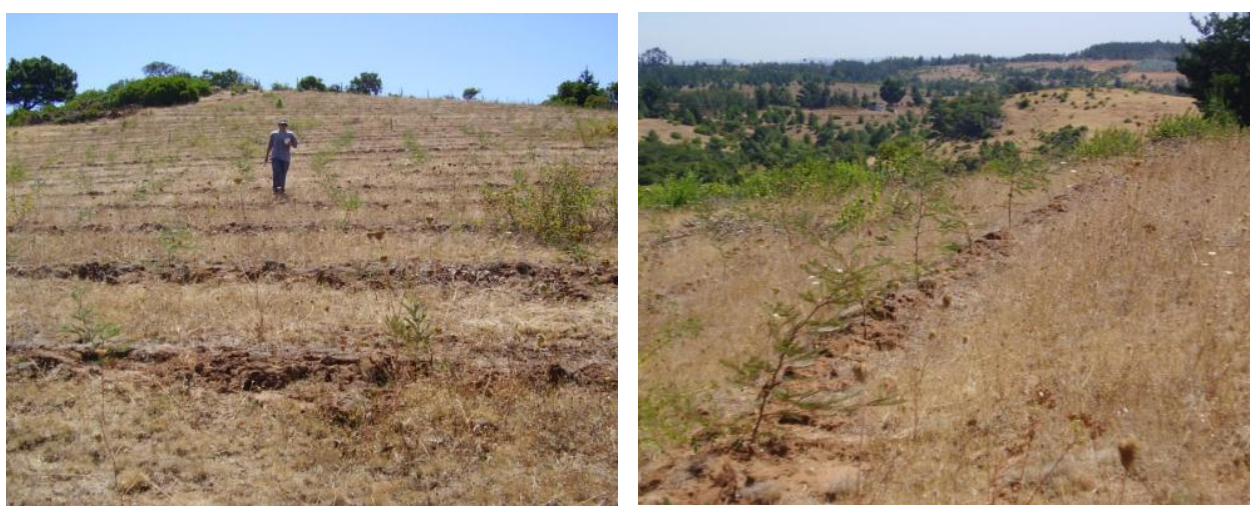

Figura $\mathrm{N}^{\circ} 4$

VISTA GENERAL ENSAYO SAN ANTONIO DE CUDA

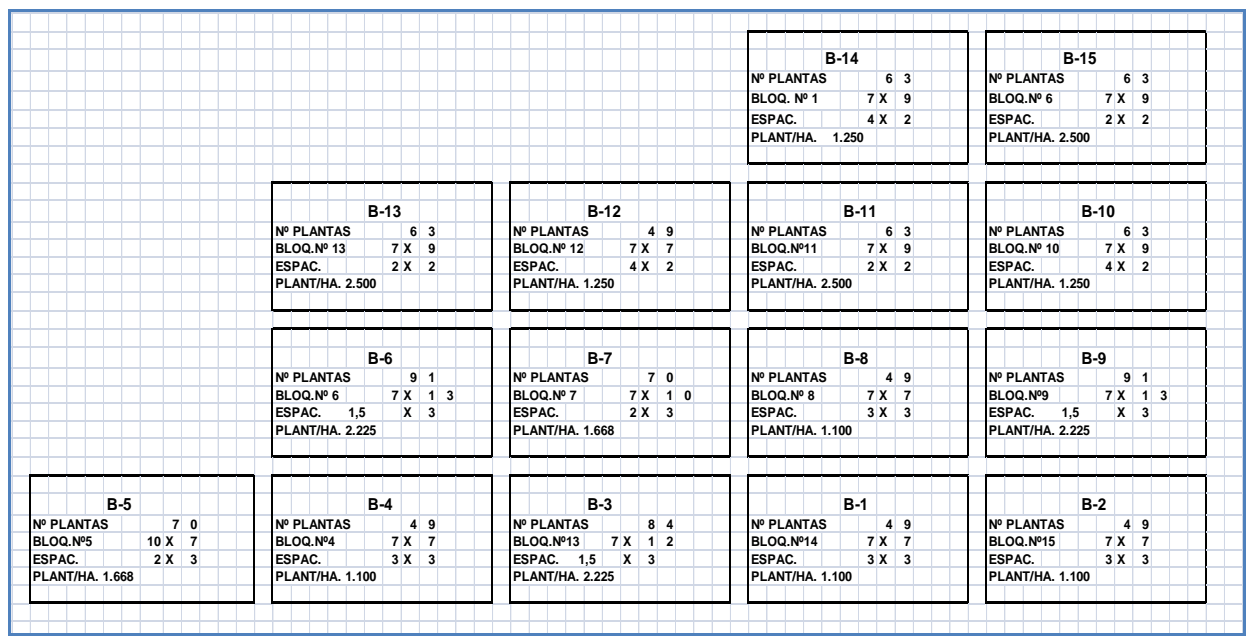

Figura $\mathrm{N}^{\circ} 5$

DISEÑO ENSAYO SAN ANTONIO DE CUDA 


\section{Variables Medidas y Análisis}

establecimiento.

La evaluación se realizó el año 2012, cuando ambos ensayos contaban con 8 años desde su

En la evaluación de los resultados se aplicó un análisis de variancia con dos factores de variación bajo control (densidad de plantación y bloques).

Cuando los resultados fueron significativos se aplicó la prueba de Tukey, para evaluar las diferencias entre los tratamientos. En ambas evaluaciones se utilizó un nivel de confianza del 95\%.

Las variables medidas en las parcelas son las siguientes:

Altura total de todos los individuos en cada parcela $(\mathrm{m})$

DAP de todos los individuos en cada parcela $(\mathrm{cm})$

Área Basal por parcela $\left(\mathrm{m}^{2} / \mathrm{ha}\right)$

Estimador de Biomasa por parcela $\left(D^{2} \times\right.$ Altura Total)

Supervivencia (\%)

\section{RESULTADOS}

Como se indicó, ambos ensayos fueron medidos el año 2012, a los 8 años de edad. Un aspecto general de estos ensayos en visión aérea se aprecia en las Figuras $N^{\circ} 6$ y $N^{\circ} 7$.

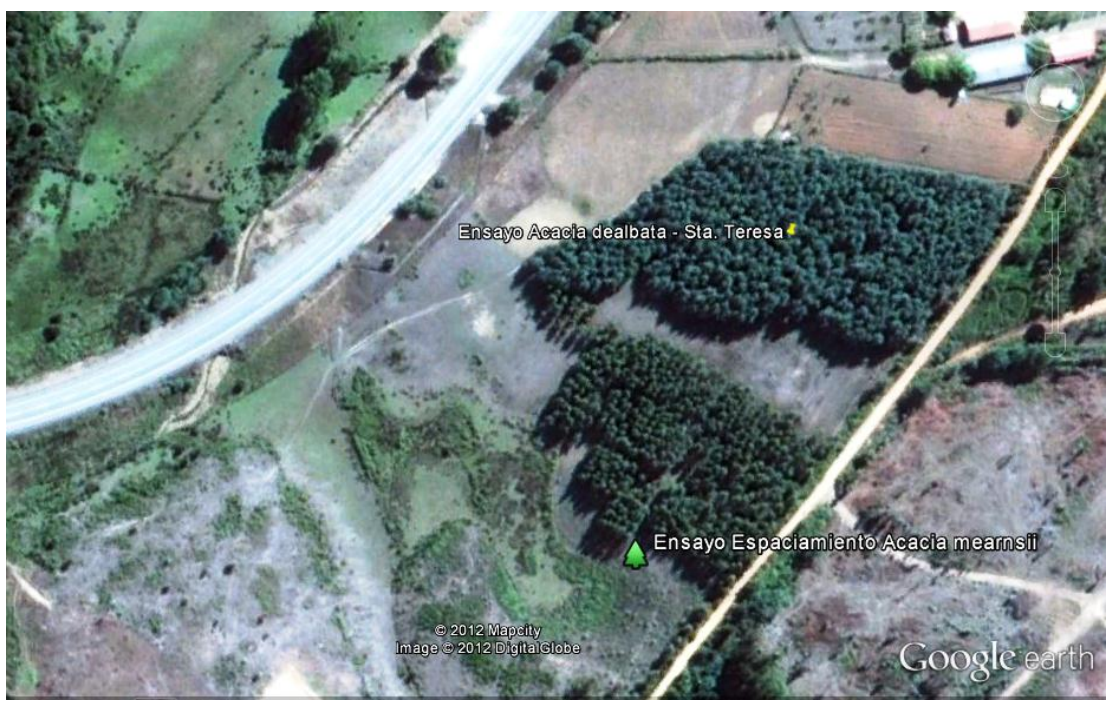

Figura $\mathbf{N}^{\circ} 6$

ASPECTO GENERAL ENSAYO SANTA TERESA, RAFAEL (2012) 


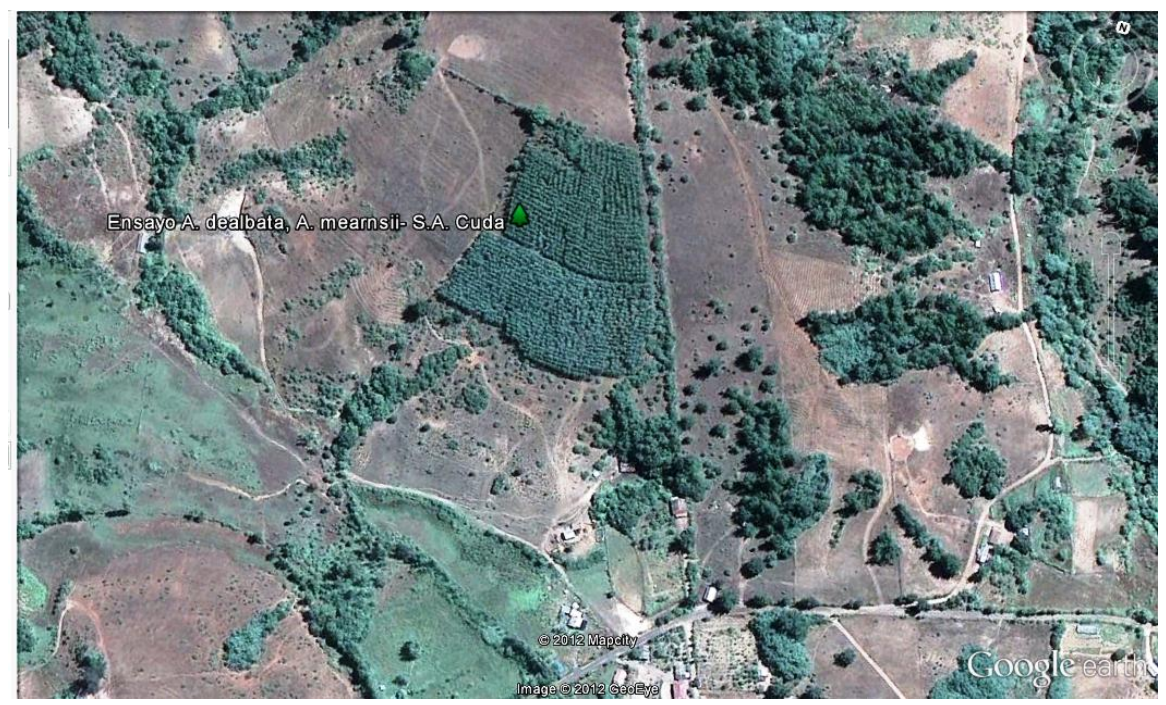

Figura $\mathrm{N}^{\circ} 7$ ASPECTO GENERAL ENSAYO SAN ANTONIO DE CUDA, FLORIDA (2012)

Los resultados generales de la medición realizada en ambos ensayos son presentados en el Cuadro $\mathrm{N}^{\circ} 2$.

La comparación del desarrollo en biomasa de Acacia mearnsii en ambos ensayos, ante la no existencia de modelos de volumen para la especie desarrollados en el país, se efectuó utilizando una variable estimadora de biomasa, que corresponde al producto del cuadrado del DAP multiplicado por la altura $\left(D^{2} H\right)$.

Una descripción del crecimiento general de Acacia mearnsii en estos ensayos se puede observar a través de la relación entre sus diámetros y las alturas observadas en los años 2005 y 2012, cuando el ensayo tenía 1 y 8 años, respectivamente. Esto se presenta en las Figuras $\mathrm{N}^{\circ} 8$ y $\mathrm{N}^{\circ} 9$.

Una primera revisión de estos antecedentes señala que en Santa Teresa se presentan mayores dimensiones en diámetro y altura, lo que se traduce en mayores volúmenes esperados.

En relación con la supervivencia de los ensayos, el ensayo de San Antonio de Cuda presentó un $96 \%$ en promedio, mientras que el ensayo de Santa Teresa fue afectado por una intervención accidental, que afectó algunas parcelas y redujo la supervivencia al $63 \%$. 
Cuadro $\mathrm{N}^{\circ} 2$

RESULTADOS GENERALES SEGÚN ESPACIAMIENTO Y ENSAYO

(8 años edad)

\begin{tabular}{|l|l|l|}
\hline \multirow{2}{*}{ Variable } & \multicolumn{2}{|c|}{ Ensayo } \\
\cline { 2 - 3 } & Santa Teresa, Rafael & San Antonio de Cuda, Florida \\
\hline
\end{tabular}

\begin{tabular}{|c|c|c|c|c|c|c|}
\hline $\begin{array}{l}\text { DAP } \\
(\mathrm{cm})\end{array}$ & & & & & & \\
\hline Tratamiento & Media & $\begin{array}{c}\text { Valor } \\
\text { Mínimo }\end{array}$ & $\begin{array}{c}\text { Valor } \\
\text { Máximo }\end{array}$ & Media & $\begin{array}{c}\text { Valor } \\
\text { Mínimo }\end{array}$ & $\begin{array}{c}\text { Valor } \\
\text { Máximo }\end{array}$ \\
\hline $3 \times 1,5$ & 14,24 & 7 & 17 & 9,88 & 4,2 & 16 \\
\hline $3 \times 2$ & 14,14 & 7,2 & 19,6 & 10,26 & 5,1 & 15 \\
\hline $3 \times 3$ & 13,26 & 9,6 & 17,6 & 11,23 & 4,7 & 16,3 \\
\hline $2 \times 2$ & 13,11 & 6,9 & 18,5 & 9,98 & 6,2 & 14,5 \\
\hline $2 \times 4$ & 12,28 & 6 & 17,3 & 11,4 & 3,5 & 16,2 \\
\hline
\end{tabular}

\begin{tabular}{|c|r|r|r|r|r|r|}
\hline $\begin{array}{c}\text { ALTURA } \\
(\boldsymbol{m})\end{array}$ & \multicolumn{10}{|c|}{} \\
\hline $\mathbf{3} \times \mathbf{1}, \mathbf{5}$ & 12,4 & 3,5 & 19,2 & 12,34 & 6,2 & 15,8 \\
\hline $\mathbf{3} \times \mathbf{2}$ & 14,14 & 5,7 & 24,3 & 12,21 & 9 & 14,6 \\
\hline $\mathbf{3} \times \mathbf{3}$ & 13,87 & 6,1 & 21,1 & 11,62 & 5,6 & 13,9 \\
\hline $\mathbf{2} \times \mathbf{2}$ & 12,13 & 4,9 & 17 & 11,33 & 7,8 & 13,5 \\
\hline $\mathbf{2} \times \mathbf{4}$ & 13,25 & 6,8 & 20,2 & 11,26 & 7,1 & 13,9 \\
\hline
\end{tabular}

\begin{tabular}{|c|r|r|r|r|r|r|}
\hline \multicolumn{1}{|c|}{$\boldsymbol{D}^{2} \boldsymbol{H}$} & \multicolumn{7}{|c|}{} \\
\hline $\mathbf{3 \times 1 , 5}$ & 2.689 & 172 & 5.419 & 1.344 & 109 & 3.748 \\
\hline $\mathbf{3} \times \mathbf{2}$ & 3.007 & 296 & 9.335 & 1.349 & 234 & 3.060 \\
\hline $\mathbf{3} \mathbf{3}$ & 2.548 & 562 & 6.071 & 1.552 & 124 & 3.667 \\
\hline $\mathbf{2} \times \mathbf{2}$ & 2.233 & 268 & 4.518 & 1.212 & 311 & 2.565 \\
\hline $\mathbf{2} \times \mathbf{4}$ & 2.358 & 244 & 5.301 & 1.567 & 87 & 3.071 \\
\hline
\end{tabular}




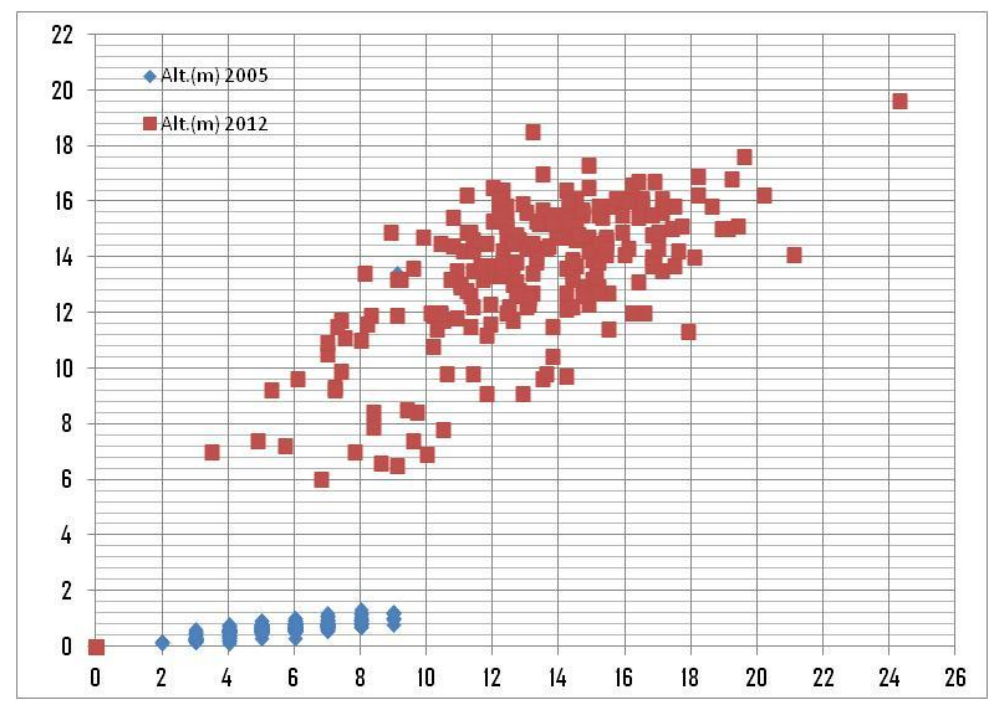

Figura $N^{\circ} 8$

RELACIÓN DIÁMETRO Y ALTURA ENSAYO SANTA TERESA, RAFAEL

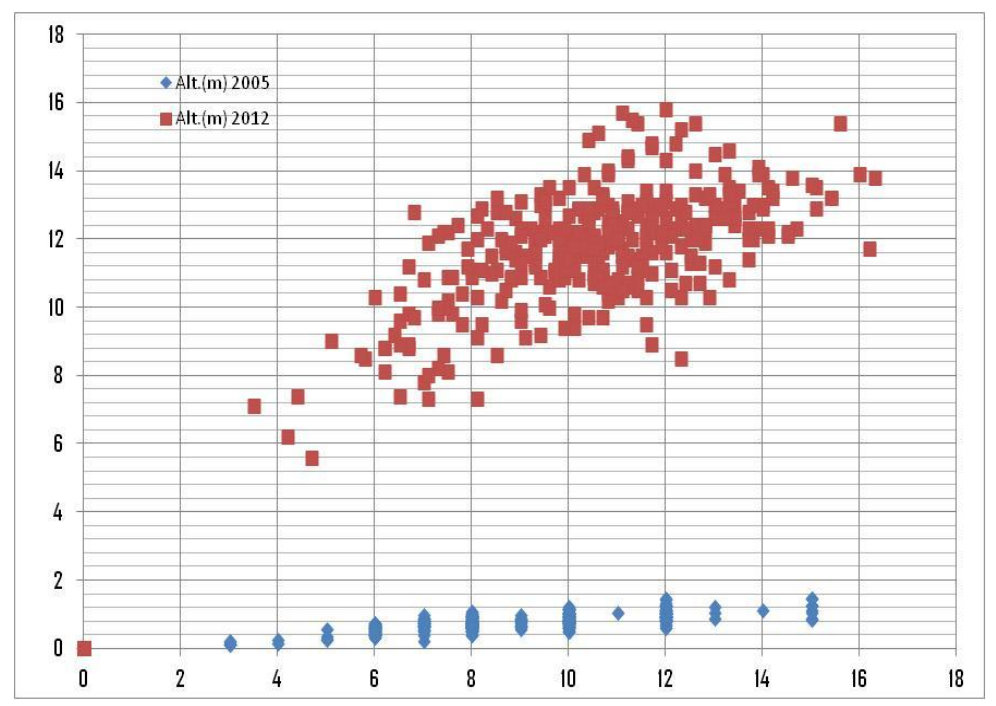

Figura $N^{\circ} 9$

RELACIÓN DIÁMETRO Y ALTURA ENSAYO SAN ANTONIO DE CUDA, FLORIDA 


\section{Desarrollo en DAP}

Los resultados obtenidos en el crecimiento del DAP, a los 8 años en cada ensayo, son presentados en el Cuadro $\mathrm{N}^{\circ} 3$ y en la Figura $\mathrm{N}^{\circ} 10$.

\section{RESULTADOS EN DAP SEGÚN ESPACIAMIENTO Y ENSAYO}

\begin{tabular}{|c|c|c|c|c|c|c|c|}
\hline \multirow{2}{*}{\multicolumn{2}{|c|}{$\begin{array}{l}\text { Espaciamiento } \\
\text { (m) (arb/ha) }\end{array}$}} & \multicolumn{2}{|l|}{$\begin{array}{c}\text { Santa Teresa } \\
\text { Rafael }\end{array}$} & \multicolumn{4}{|c|}{$\begin{array}{l}\text { San Antonio de Cuda } \\
\text { Florida }\end{array}$} \\
\hline & & \multicolumn{6}{|c|}{$\begin{array}{l}\text { Medias } \\
(\mathrm{cm})\end{array}$} \\
\hline $2 \times 4$ & $(1.250)$ & $12,28 \quad A$ & & 11,4 & & & $C$ \\
\hline $2 \times 2$ & $(2.500)$ & $13,11 \quad A$ & $\mathrm{~B}$ & 9,98 & A & & \\
\hline $3 \times 3$ & (1.111) & $13,26 \quad A$ & B & 11,23 & & B & C \\
\hline $3 \times 2$ & $(1.667)$ & 14,14 & B & 10,26 & $A$ & $B$ & \\
\hline $3 \times 1,5$ & (2.222) & 14,24 & $B$ & 9,88 & $A$ & & \\
\hline
\end{tabular}

Letras distintas indican diferencias significativas $(p<=0,05)$

El análisis de variancia realizado permite detectar una diferencia significativa entre los diámetros medios de las diferentes densidades a los 8 años de edad, en ambos ensayos, sin haber existido diferencias significativas entre bloques. Se destaca el mayor valor registrado en el ensayo Santa Teresa.

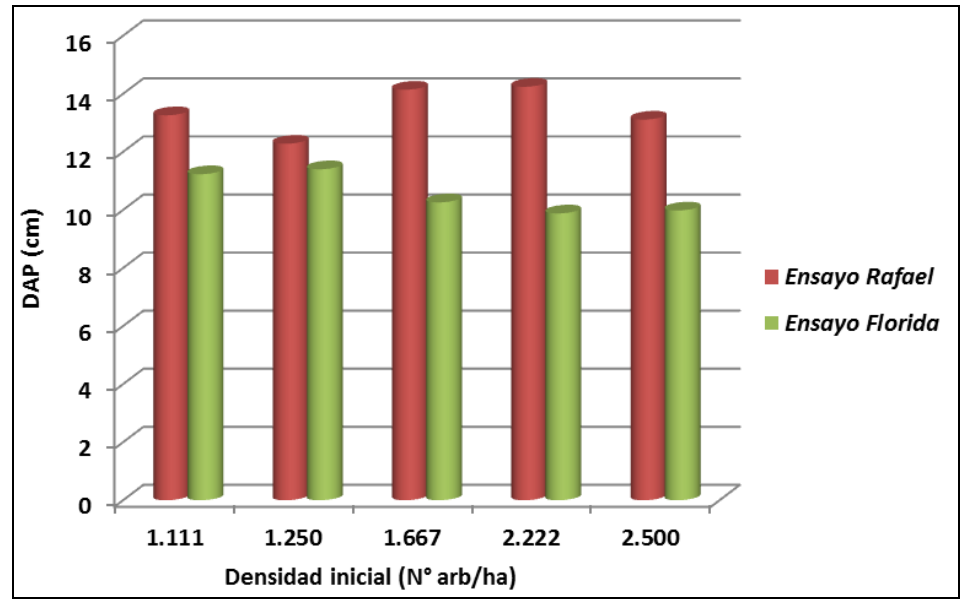

\section{Figura $\mathrm{N}^{\circ} 10$ \\ COMPARACIÓN DE LOS RESULTADOS EN DAP \\ SEGÚN ESPACIAMIENTO Y ENSAYO \\ (8 años edad)}

Al momento de la evaluación los doseles de ambos ensayos estaban en general cerrados, sin embargo es necesario señalar que en el caso del ensayo Rafael, algunas parcelas sufrieron una alteración productos de trabajos realizados por el propietario en el terreno. Ello implicó que 2 parcelas, de las 15 en total, presentaran una supervivencia cercana al $40 \%$ (2 x 2 y 2 x $4 \mathrm{~m}$ ), lo que llevó a que el promedio general del ensayo llegara al $65 \%$. 
Como antecedente es posible señalar que en el ensayo Florida, la supervivencia promedio fue del $96 \%$, donde el menor valor se obtuvo en la parcela con el espaciamiento de $3 \times 3$ m con un valor del $89 \%$.

En las pruebas de comparación, los tratamientos con mayor densidad de árboles en el ensayo de Rafael presentan los mayores diámetros y se diferencian entre sí, pero se debe considerar lo señalado previamente respecto de la alteración de algunas de las parcelas. En el ensayo de Florida, los tratamientos con menor espaciamiento presentan un diámetro medio menor, con diferencia estadística entre ellos.

El comportamiento observado coincide con los resultados obtenidos en ensayos similares instalados en Argentina, de la misma especie y de otras edades (Correa et al., 2011).

Los resultados señalan que las densidades más bajas han presentado en general mayores tasas de crecimiento.

\section{Desarrollo en Altura}

Los resultados obtenidos en el crecimiento en altura a los 8 años en cada ensayo son resumidos en el Cuadro $\mathrm{N}^{\circ} 4$ y la Figura $\mathrm{N}^{\circ} 11$.

\section{RESULTADOS EN ALTURA SEGÚN ESPACIAMIENTO Y ENSAYO}

\begin{tabular}{|c|c|c|c|c|c|c|c|c|}
\hline \multirow{2}{*}{\multicolumn{2}{|c|}{$\begin{array}{l}\text { Espaciamiento } \\
\text { (m) (arb/ha) }\end{array}$}} & \multicolumn{3}{|c|}{$\begin{array}{c}\text { Santa Teresa } \\
\text { Rafael }\end{array}$} & \multicolumn{4}{|c|}{$\begin{array}{c}\text { San Antonio de Cuda } \\
\text { Florida }\end{array}$} \\
\hline & & \multicolumn{7}{|c|}{$\begin{array}{l}\text { Medias } \\
(\mathrm{m})\end{array}$} \\
\hline $2 \times 4$ & $(1.250)$ & 13,25 & A & $B$ & 11,26 & $A$ & & \\
\hline $2 \times 2$ & $(2.500)$ & 12,13 & A & & 11,33 & $A$ & & \\
\hline $3 \times 3$ & $(1.111)$ & 13,87 & A & $B$ & 11,62 & $A$ & $B$ & \\
\hline $3 \times 2$ & $(1.667)$ & 14,14 & & $B$ & 12,21 & & $B$ & C \\
\hline $3 \times 1,5$ & (2.222) & 12,40 & $A$ & $B$ & 12,34 & & & C \\
\hline
\end{tabular}

Letras distintas indican diferencias significativas $(p<=0,05)$

El análisis de variancia realizado permite detectar una diferencia significativa entre la altura media en ambos ensayos. No se observaron diferencias significativas entre bloques. Se destaca nuevamente el mayor valor registrado en el ensayo en Rafael. 


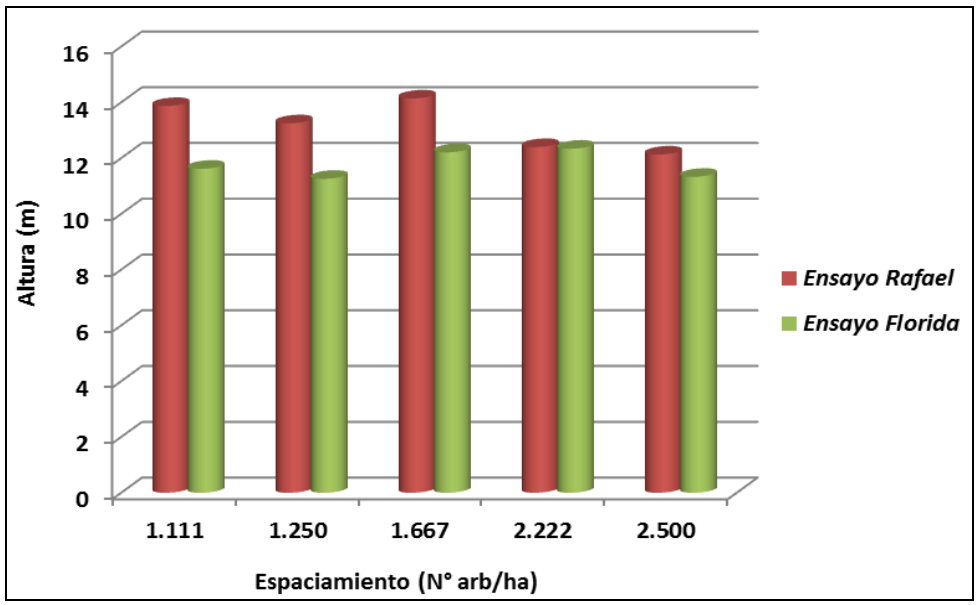

Figura $\mathrm{N}^{\circ} 11$

COMPARACIÓN DE LOS RESULTADOS EN ALTURA SEGÚN DENSIDAD INICIAL Y ENSAYO

(8 años edad)

En el ensayo de Rafael los tratamientos con menor densidad de árboles presentan las mayores alturas medias no siendo en general, significativamente diferentes entre sí. En el ensayo de Florida los resultados son similares.

\section{Desarrollo en Área Basal}

Los resultados obtenidos para el área basal a la edad de 8 años, según cada espaciamiento utilizado, se presentan en el Cuadro $N^{\circ} 5$ y Figura $N^{\circ} 12$.

\section{Cuadro $\mathrm{N}^{\circ} 5$ \\ RESULTADOS EN ÁREA BASAL SEGÚN ESPACIAMIENTO Y ENSAYO}

\begin{tabular}{|c|c|c|c|c|c|c|c|c|}
\hline \multirow{2}{*}{\multicolumn{2}{|c|}{$\begin{array}{l}\text { Espaciamiento } \\
\text { (m) (arb/ha) }\end{array}$}} & \multicolumn{3}{|c|}{$\begin{array}{c}\text { Santa Teresa } \\
\text { Rafael }\end{array}$} & \multicolumn{4}{|c|}{$\begin{array}{c}\text { San Antonio de Cuda } \\
\text { Florida }\end{array}$} \\
\hline & & \multicolumn{7}{|c|}{$\begin{array}{l}\text { Medias } \\
\left(\mathrm{m}^{2} / \mathrm{ha}\right)\end{array}$} \\
\hline $2 \times 4$ & $(1.250)$ & 8,88 & A & & 12,50 & A & & \\
\hline $2 \times 2$ & $(2.500)$ & 20,43 & C & & 19,33 & & & $C$ \\
\hline $3 \times 3$ & $(1.111)$ & 9,74 & A & & 10,67 & A & & \\
\hline $3 \times 2$ & (1.667) & 16,69 & $B$ & & 13,77 & A & B & \\
\hline $3 \times 1,5$ & (2.222) & 24,48 & & $D$ & 17,80 & & B & C \\
\hline
\end{tabular}

Letras distintas indican diferencias significativas $(p<=0,05)$

El análisis de variancia realizado permite detectar una diferencia significativa en área basal en ambos ensayos. Se destaca nuevamente los mayores valores registrados en el ensayo en Rafael.

Es necesario señalar que el valor del área basal en el ensayo de Rafael está afectado por la intervención que sufrió el ensayo y que significó una menor supervivencia de árboles (63\%). Esto afecto especialmente a las parcelas con menor densidad. En general el comportamiento del área basal fue similar en ambos ensayos. 


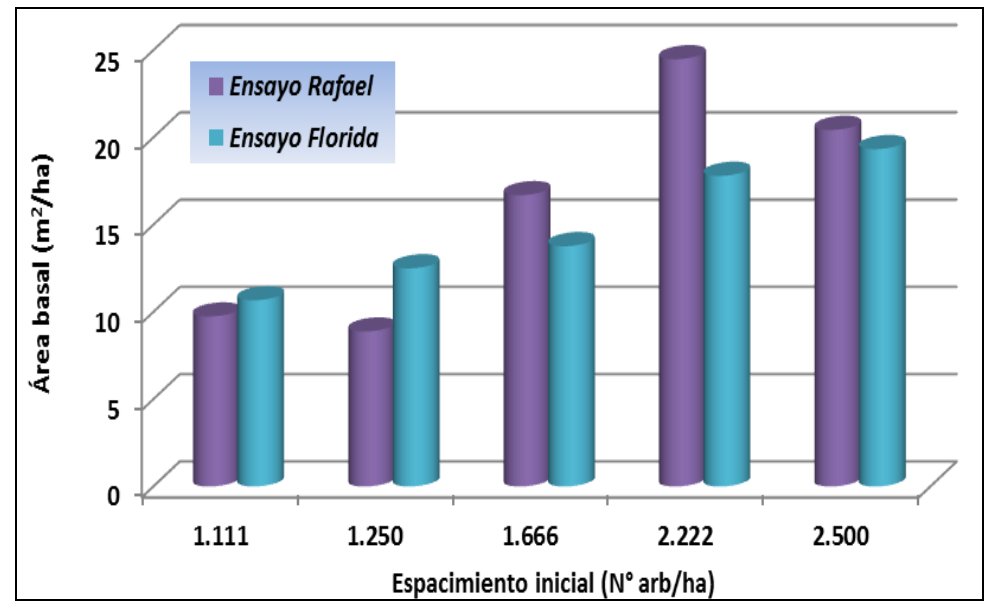

Figura $\mathrm{N}^{\circ} 12$

COMPARACIÓN DE LOS RESULTADOS EN ÁREA BASAL SEGÚN ESPACIAMIENTO Y ENSAYO

(8 años edad)

En el ensayo de Rafael los tratamientos con mayor densidad en general presentaron un mayor valor en área basal, siendo significativamente diferentes entre sí. En el ensayo de Florida los resultados son similares.

Los resultados señalan que las densidades más altas han presentado en general mayores tasas de crecimiento en área basal.

\section{Desarrollo en Biomasa}

Tal como se señaló previamente, para poder hacer una estimación y comparación del desarrollo en biomasa de Acacia mearnsii en ambos ensayos, se utilizó una variable estimadora de biomasa, la que corresponde al producto del cuadrado del DAP multiplicado por la altura $\left(D^{2} H\right)$.

Los resultados obtenidos para la estimación y comparación de la biomasa a la edad de 8 años desde cada ensayo, se presentan en el Cuadro $N^{\circ} 6$ y Figura $N^{\circ} 13$.

Cuadro $\mathrm{N}^{\circ} 6$

RESULTADOS EN ESTIMACIÓN DE BIOMASA SEGÚN ESPACIAMIENTO Y ENSAYO

\begin{tabular}{|c|c|c|c|c|c|c|c|}
\hline \multirow{2}{*}{\multicolumn{2}{|c|}{$\begin{array}{l}\text { Espaciamiento } \\
\text { (m) (arb/ha) }\end{array}$}} & \multicolumn{3}{|c|}{$\begin{array}{c}\text { Santa Teresa } \\
\text { Rafael }\end{array}$} & \multicolumn{3}{|c|}{$\begin{array}{c}\text { San Antonio de Cuda } \\
\text { Florida }\end{array}$} \\
\hline & & \multicolumn{6}{|c|}{$\begin{array}{l}\text { Medias } \\
\left(D^{2} H\right)\end{array}$} \\
\hline $2 \times 4$ & $(1.250)$ & $2.357,88$ & A & B & $1.566,60$ & & $B$ \\
\hline $2 \times 2$ & $(2.500)$ & $2.233,35$ & A & & $1.211,84$ & A & \\
\hline $3 \times 3$ & (1.111) & $2.547,67$ & $A$ & $B$ & $1.551,75$ & & $B$ \\
\hline $3 \times 2$ & $(1.667)$ & $3.006,87$ & & $B$ & $1.348,97$ & $A$ & $B$ \\
\hline $3 \times 1,5$ & (2.222) & $2.689,34$ & $A$ & B & $1.343,69$ & A & B \\
\hline
\end{tabular}

Letras distintas indican diferencias significativas $(p<=0,05)$ 
El análisis de variancia realizado permite detectar una diferencia significativa en la estimación de biomasa en ambos ensayos. Se destaca nuevamente los mayores valores registrados en el ensayo en Rafael.

En general el comportamiento del estimador de biomasa fue similar al presentado para el caso del área basal en ambos ensayos.

En el ensayo de Rafael los tratamientos con 1.667 arb/ha presentan un mayor valor en la estimación de biomasa, siendo significativamente diferentes con el resto de las densidades ensayadas. Este espaciamiento junto al de 2.222 arb/ha presentaron los mayores valores. En el ensayo de Florida los mayores resultados en biomasa se obtuvieron en los espaciamientos de 1.250 y $1.111 \mathrm{arb} / \mathrm{ha}$, no siendo en general estadísticamente distintos del resto de las densidades.

Los resultados señalan que las densidades intermedias han presentado en general mayores tasas de crecimiento en área basal.

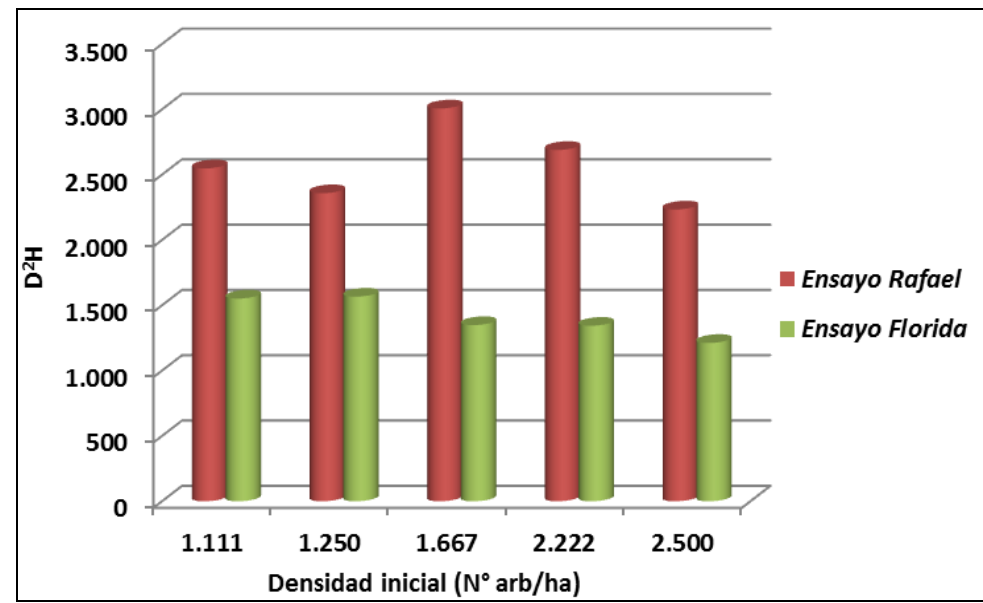

\section{Figura $N^{\circ} 13$ \\ COMPARACIÓN DE LOS RESULTADOS EN ESTIMACIÓN DE BIOMASA SEGÚN DENSIDAD INICIAL Y ENSAYO \\ (8 años edad)}

\section{DISCUSIÓN}

Los resultados obtenidos a los 8 años desde los 2 ensayos evaluados indican un efecto significativo del espaciamiento inicial sobre el crecimiento en diámetro y el área basal. Además, se observa una diferencia entre los valores obtenidos a partir de cada ensayo, señalando un efecto del sitio sobre el valor de las variables resultantes.

Los valores registrados son similares a los señalados por la bibliografía, la que indica que Acacia mearnsii es un arbusto grande o un árbol pequeño, comúnmente de 6 a $10 \mathrm{~m}$ de altura, pero puede alcanzar hasta $20 \mathrm{~m}$ (Kannegiesser, 1990).

Correa et al., 2011, señalan que en un ensayo de 3 años no se observó diferencias significativas en el volumen sin corteza de madera, señalando que esta situación sería debida a la temprana edad en que se realiza esta evaluación. Agregan que existiría una relación inversa en función de las distancias de plantación, lo que indica que a menor espaciamiento se genera una mayor producción de volumen. 
En Brasil, más específicamente en el Estado de Río Grande do Sul, donde existen plantaciones con Acacia mearnsii, una plantación de ocho años produce aproximadamente $200 \mathrm{~m}^{3}$ de madera y 16 toneladas de corteza (Higa et al, 1998; Pinilla y Gutiérrez, 2000). En este país, en los mejores suelos, es usual utilizar un menor espaciamiento inicial a fin de obtener un mayor volumen de madera, empleando espaciamientos de $3 \times 3$ metros a 2,5 $\times 3$ metros. En relación con las técnicas de establecimiento, estas son simples. Normalmente el agricultor usa el fuego para limpiar los campos, consiguiendo con esto romper la latencia de las semillas de acacia y favorecer su germinación. Posteriormente, dependiendo de la magnitud de la regeneración, puede manejarla o realizar una plantación. El uso de fertilizantes es una práctica eventual (Pinilla y Gutiérrez, 2000).

En este mismo país, Guimarães et al. (2000) señalan que la especie en rotaciones de 7 años, utilizando espaciamientos de $3 \times 1,3 \mathrm{~m}$, presenta una altura media de 15,8 m, un DAP de $12,4 \mathrm{~cm}$ y un área basal de $19,2 \mathrm{~m}^{2} / \mathrm{ha}$.

El rendimiento típico de una plantación comercial bien manejada de 10-11 años en Natal, Sudáfrica, es de 21 t/ha de corteza seca y 112 t/ha de madera seca al aire. En el más fresco Transvaal los mismos resultados corresponden a 16,6 y $74,8 \mathrm{t} / \mathrm{ha}$, respectivamente. A esta edad los árboles miden $17,4 \mathrm{~m}$ de altura y $14,5 \mathrm{~cm}$ de diámetro en Natal, y $14,4 \mathrm{~m}$ y $13,4 \mathrm{~cm}$ en Transvaal (Stubbing y Shonau, 1982, citados por Kannegiesser, 1990). Estos resultados también son coincidentes con los obtenidos en estos ensayos en Chile. En sitios apropiados y donde los árboles son fertilizados es posible obtener un incremento medio anual de $15-25 \mathrm{~m}^{3} / \mathrm{ha}$ de madera a los 7 - 10 años (Turnbull, 1986).

Los resultados obtenidos también son comparables con los reportados desde Fukuoka (Japón), donde plantaciones no manejadas, que se establecieron en suelos derivados de granitos, presentaron alturas de $7 \mathrm{~m}$ a los 3 años; $13 \mathrm{~m}$ a los 6 años y $15 \mathrm{~m}$ a los 8 años. Los DAP medios de estos rodales fueron $0,6 \mathrm{~cm}$ al año; $5 \mathrm{~cm}$ a los 3 años y $13 \mathrm{~cm}$ a los 8 años. A los 10 años y con $1.500 \mathrm{arb} / \mathrm{ha}$ al final de la rotación se produjeron $12 \mathrm{t} / \mathrm{ha}$ de corteza y $130 \mathrm{~m}^{3} / \mathrm{ha}$ de madera. Con un manejo apropiado se puede obtener alrededor de $200 \mathrm{~m}^{3} /$ ha de madera a los $10-11$ años (Kannegiesser, 1990).

Kannegiesser (1990) señala que la plantación de esta especie se puede realizar a espaciamientos de $2 \times 2 \mathrm{~m} ; 2,5 \times 2,5 \mathrm{~m} ; 3 \times 1,3 \mathrm{~m}$ y $2 \times 3 \mathrm{~m}$. Los espaciamientos mayores se practican en aquellas áreas donde se realizan cultivos interfilas y además se permite el uso de implementos mecánicos. Estos espaciamientos originan rodales con densidades entre 2.500 y 1.700 árboles por hectárea. Agrega que la mejor corteza, a los 7 - 10 años de edad, se produce en rodales cuyas densidades fluctúan entre 1.200 y 1.500 árboles por hectárea.

En parcelas de introducción de especies ubicadas en la Hacienda Rucamanqui (Región del Bio Bio), de propiedad de Forestal y Agrícola Monteáguila SA, el desarrollo de Acacia mearnsii mostró ser sobresaliente (INFOR, 1995). La Empresa realizó ensayos de pulpaje y sus características han resultado ser apropiadas para la producción de pulpa en mezcla con Eucalyptus $s p$ (Pinilla, 2000).

Paralelamente, INFOR ha desarrollado en otros estudios relaciones funcionales estáticas y dinámicas a nivel local para Acacia mearnsii, intentando evaluar esquemas silviculturales y de manejo (INFOR, 1998; 1999). Esta misma investigación menciona que no sería posible manejar plantaciones bajo el esquema de monte bajo, debido a la escasa retoñación de tocones. Los crecimientos medios anuales en volumen se estimaron cercanos a $5 \mathrm{~m}^{3} / \mathrm{ha} / \mathrm{año}$, presentando una respuesta inmediata al raleo. Finalmente, se concluye que esta especie, debido a sus características de crecimiento y su diversidad de usos, es una interesante alternativa productiva para sectores de la región del secano interior hacia el sur.

Pinilla et al. (2004), reportan un ensayo de especies y procedencias en que los resultados señalaron diferencias significativas entre las especies; Acacia dealbata, Acacia melanoxylon y Acacia mearnsii (Cuadro $\mathrm{N}^{\circ} 7$ ). En general estos datos coinciden con los obtenidos desde los ensayos evaluados. 


\section{Cuadro $\mathrm{N}^{\circ} 7$ \\ PARÁMETROS OBTENIDO EN UN ENSAYO CON ACACIAS A LOS 9 AÑOS DE EDAD}

\begin{tabular}{|c|c|c|c|}
\hline Especies & $\begin{array}{l}\text { DAP } \\
\text { (cm) }\end{array}$ & $\begin{array}{l}\text { Altura } \\
(\mathrm{m})\end{array}$ & $\begin{array}{c}\text { Supervivencia } \\
(\%)\end{array}$ \\
\hline Acacia dealbata & 16,5 & 18,4 & 90 \\
\hline Acacia mearnsii & 12,5 & 16,1 & 87 \\
\hline Acacia melanoxylon & 9,7 & 11,6 & 85 \\
\hline
\end{tabular}

En general no existe mucha información en cuanto al efecto de la densidad de plantación sobre el crecimiento de Acacia mearnsii. En los ensayos evaluados, los tratamientos con mayor densidad de árboles en el ensayo de Rafael presentan los mayores diámetros y se diferencian entre sí, mientras que en el ensayo de Florida, los tratamientos con menor espaciamiento presentan un diámetro medio menor, con diferencian estadística entre ellos.

En el caso de una plantación de $A$. mearnsii, establecida para obtener corteza y madera, los esquemas de raleo deben orientarse a obtener la cantidad o calidad de ambos productos o para obtener el máximo rendimiento de uno a expensas del otro (Pinilla y Gutiérrez, 2000). Estos esquemas de raleo son importantes en el caso que se requiera manejar rodales naturales de la especie, de modo de que a través de 2 o 3 intervenciones se llegue al número de árboles final deseado.

El comportamiento observado coincide en cierta medida y en especial en el ensayo Florida, con los resultados obtenidos en ensayos instalados en Argentina, similares de la misma especie y de otras edades (Correa et al., 2011), donde se encontró que las distancias de plantación presentaron un efecto significativo sobre el crecimiento en diámetro medio aritmético, evidenciándose que a mayor distancia de plantación mayor diámetro medio.

Los resultados señalan que las densidades más bajas han presentado en general mayores tasas de crecimiento en biomasa, con la excepción del ensayo Rafael que fue afectado por la cosecha de árboles efectuada por el propietario alterando el diseño. Sin embargo, a pesar de la pérdida de árboles, los resultados obtenidos señalan un interesante potencial productivo de la especie en esa zona.

En el ensayo de Rafael los tratamientos con mayor densidad en general presentaron un mayor valor en área basal, siendo significativamente diferentes entre sí. En el ensayo de Florida los resultados son similares. Estos resultados señalan que las densidades más altas han presentado en general mayores tasas de crecimiento en área basal.

Igual situación se observó al analizar el estimador de biomasa; a una menor distancia de plantación se obtiene una mayor producción. Destacan nuevamente los mayores valores registrados en el ensayo en Rafael. En general el comportamiento del estimador de biomasa fue similar al presentado para el caso del área basal en ambos ensayos.

Un aspecto interesante de señalar es que, dado que la madera de Acacia mearnsii proporciona una excelente leña, con un peso específico de 0,70 a $0,85 \mathrm{~g} / \mathrm{cm}^{3}$ y un poder calorífico de 14.630 a $16.720 \mathrm{~kJ} / \mathrm{kg}$ (Kannegiesser, 1990), los resultados de ensayos de espaciamiento inicial pueden ser también utilizados si la opción es la generación de biomasa para energía.

\section{CONCLUSIONES}

Las necesidades de información para la adopción de nuevas especies forestales para su uso en el sector forestal nacional, en este caso especies del género Acacia, requiere de información de diversos aspectos, siendo relevantes aquellos asociados a su adaptación a diferentes áreas en el país y las estimaciones de crecimiento y rendimiento. Ello implica analizar el 
comportamiento de las principales variables de rodal, intentando abarcar un rango de edades, densidades y calidades de sitio.

El trabajo desarrollado por INFOR con estas especies es un primer esfuerzo por lograr información confiable al respecto y se ha complementado con estudios de las propiedades de su madera y con los primeros avances en un programa de mejoramiento genético.

Este estudio realizado por INFOR es el primer trabajo sobre el efecto del espaciamiento inicial en Acacia mearnsii en el país y abarcó dos áreas geográficas en la región del Bio Bio, Los resultados son satisfactorios en cuanto al crecimiento de la especie y constituyen una primera e información sobre rendimientos y crecimientos, antecedentes de importancia para la toma de decisiones por parte de interesados en el cultivo de estas especies forestales alternativas a las tradicionalmente empleadas.

Como conclusiones preliminares se puede señalar las siguientes:

Acacia mearnsii parece una interesante alternativa para los forestadores e industrias dedicadas a la producción de pulpa y madera aserrada.

Acacia mearnsii se presenta como una especie que justifica mayor investigación por su adaptabilidad y características de crecimiento, y por los posibles productos a obtener desde ella (madera, biomasa, taninos a partir de su corteza).

En el ensayo de Rafael se registra un valor promedio a los 8 años de edad de $13,4 \mathrm{~cm}$ y 13,2 $\mathrm{m}$ en DAP y altura, respectivamente, y los valores máximos medios registrados para estas variables son de $18 \mathrm{~cm}$ y $20,4 \mathrm{~m}$, respectivamente.

En el ensayo en Florida se registra un valor promedio a los 8 años de edad de 10,6 cm y $11,8 \mathrm{~m}$ en DAP y altura, respectivamente, y los valores máximos medios alcanzan $15,6 \mathrm{~cm}$ y 14,3 $\mathrm{m}$, respectivamente.

Las áreas basales alcanzan a $15,5 \mathrm{~m}^{2} /$ ha en el ensayo en Rafael y $14,8 \mathrm{~m}^{2} / \mathrm{ha}$ en el establecido en Florida.

La supervivencia fue de un $96 \%$ en el ensayo de Florida y un $63 \%$ en el ensayo de Rafael, este último afectado por una intervención ajena al diseño. Este factor afectó necesariamente los resultados del ensayo en Rafael, sin embargo, los valores obtenidos desde esta unidad confirman el potencial de la especie.

Se destaca en general en los ensayos los mayores valores registrados en la unidad establecida en Tomé, sector Rafael, que ponen en evidencia un efecto de sitio en los resultados obtenidos.

La densidad inicial presentó un efecto significativo sobre el crecimiento en DAP, evidenciándose que a mayor distancia de plantación mayor diámetro medio.

Los resultados señalan que las densidades más altas han presentado en general mayores tasas de crecimiento en área basal e igual situación se observó al analizar el estimador de biomasa, en el cual a una menor distancia de plantación se obtiene una mayor producción.

Estos resultados son de interés en función de los productos objetivo que se planteen; para productos menores como pulpa o energía los espaciamientos estrechos aparecen como más convenientes en rotaciones cortas.

Es necesario seguir ensayando procedencias y progenies de esta especie en otras áreas geográficas, con el objetivo aumentar la cobertura de la información y seleccionar material apropiado para la forestación y para avanzar hacia un mejoramiento genético. 
Se debe dar seguimiento por más tiempo a estos ensayos de espaciamiento y establecer otros en diferentes áreas geográficas con el fin de permitir el desarrollo de funciones de crecimiento para la especie en diferentes sitios.

\section{REFERENCIAS}

Correa, M.; Toloza, R.; Pereyra, L.; Silva, F. y Friedl, R., 2011. Resultados iniciales de un ensayo de acacia negra (Acacia mearnsii De Wild) en la provincia de Misiones. XIII Jornadas Técnicas Forestales y Ambientales. Facultad de Ciencias Forestales, UNaM - EEA Montecarlo, INTA. El Dorado, Misiones, Argentina.

Guimarães, C.; Spathelf, P.; Schneide, P. y Coelho, L., 2000. Curvas de altura-diâmetro de acácia negra (Acacia mearnsii de Wild). Cienc. Rural vol.30 no.3 Santa Maria May/June 2000.

Higa, A. R.; Dedecek, R. A.; Dos Santos, A. F.; Stein, P. P. y Simon, A. A., 1998. Desarrollo de sistemas de producción para acacia negra (Acacia mearnsii De Wild.). Resumen.

INFOR, 1995. Investigación silvicultura de Acacia melanoxylon. (Informe de avance 1995). INFOR - CORFO. Concepción.

INFOR, 1998. Incorporación de especies del género Acacia a la producción forestal chilena. Formulario de presentación proyecto FDI. INFOR-CORFO. Santiago 150 p.

INFOR, 1999. Incorporación de especies del género Acacia a la producción forestal chilena. Informe de avance. INFOR - CORFO. Concepción. 126 p.

Kannegiesser, U., 1990. Apuntes sobre algunas acacias australianas: 1. Acacia mearnsii De Willd. Ciencia e Investigación Forestal, 4 (2):198-212.

Pinilla, J. C., 2000. Descripción y antecedentes básicos sobre Acacia dealbata, A. melanoxylon y A. mearnsii. Informe Técnico №147. INFOR-CORFO. 49p.

Pinilla, J. C. y Gutiérrez, B., 2000. Reporte de la Visita a EMBRAPA, Universidades y Empresas de Brasil. Documento de Proyecto FDI Incorporación De Especies Del Género Acacia A La Producción Forestal Chilena. Concepción, Octubre 2000. 48p.

Pinilla, J. C.; Molina M. P. y Gutiérrez, B., 2004. Acacia en el Desarrollo Forestal Productivo de Chile: Avances De Investigación. En: Actas Seminario Diversificación Forestal: Nuevas opciones, Nuevos productos, Nuevos mercados. INFOR. Villarrica, Chile. 13-15 Octubre 2004

Pinilla, J. C.; Molina, M. P.; Hernández, G.; Barros, S.; Ortiz, O. y Navarrete, M., 2011. Avances de la investigación con especies del género Acacia en Chile. Informe Técnico Nº179. Instituto Forestal, Sede Bio Bio, Chile.

NAS, 1980. Firewood crops. Shrubs and tree species for energy production. Vol. 1. National Academy of Sciences. Washington D.C. National Academy Press. 237 p.

Searle, S. D., 2000. Black Wattle (Acacia mearnsii) for farm forestry. Department of Natural Resource and Environment. Victoria. 18p.

Turnbull, J. W., 1986. Multipurpose Australian trees and shrubs. Lesser known species for fuelwood and agroforestry: ACIAR Monograph Num. 1, 316 p. 
